\title{
Sumatriptan does not change calcitonin gene-related peptide in the cephalic and extracephalic circulation in healthy volunteers
}

\author{
Jakob Møller Hansen · Jesper Petersen • \\ Troels Wienecke · Karsten Skovgaard Olsen . \\ Lars Thorbjørn Jensen · Messoud Ashina
}

Received: 19 December 2008/ Accepted: 20 January 2009/Published online: 6 March 2009

(C) Springer-Verlag 2009

\begin{abstract}
Triptans are effective and well tolerated in acute migraine management but their exact mechanism of action is still debated. Triptans might exert their antimigraine effect by reducing the levels of circulating calcitonin gene-related peptide (CGRP). To examine this question, we examined whether sumatriptan modulate the baseline CGRP levels in vivo, under conditions without trigeminovascular system activation. We sampled blood from the internal and external jugular, the cubital veins, and the radial artery before and after administration of subcutaneous sumatriptan in 16 healthy volunteers. Repeatedmeasure ANOVA showed no interaction between catheter and time of sampling and thus no significant difference in CGRP between the four catheters $(P=0.75)$. CGRP did not change over time in the four compartments $(P>0.05)$. The relative changes in CGRP between baseline and maximal sumatriptan concentration did not differ between the four vascular compartments $(P=0.49)$. It was found
\end{abstract}

J. M. Hansen $(\varangle) \cdot$ T. Wienecke $\cdot$ M. Ashina

Danish Headache Center and Department of Neurology,

Faculty of Health Sciences, Glostrup Hospital,

University of Copenhagen, Nordre Ringvej 57,

Bolig 23-24, Glostrup, 2600 Copenhagen, Denmark

e-mail: jmh@dadlnet.dk

URL: http://www.danishheadachecenter.com

J. Petersen - K. S. Olsen

Department of Anesthesia and Intensive Care,

Faculty of Health Sciences, Glostrup Hospital,

University of Copenhagen, Glostrup,

2600 Copenhagen, Denmark

\section{T. Jensen}

Department of Clinical Physiology and Nuclear Medicine,

Faculty of Health Sciences, Glostrup Hospital,

University of Copenhagen, Glostrup,

2600 Copenhagen, Denmark that Sumatriptan did not change the levels of circulating CGRP in the intra or extracerebral circulation in healthy volunteers. This speaks against a direct CGRP-reducing effect of sumatriptan in vivo in humans when the trigemino vascular system is not activated.

Keywords Calcitonin gene-related peptide · Migraine · Biomarker · Triptan · Experimental human model

\section{Introduction}

Migraine is a neurovascular headache [1], thought to arise from a primary brain dysfunction, leading to activation and sensitisation in the trigeminovascular system [2], and the release of vasoactive neuropeptides such as calcitonin gene-related peptide (CGRP) [3, 4]. CGRP is involved in the migraine pathogenesis [5], and CGRP antagonism is a promising new target in the treatment of migraine [6].

The triptans, $5-\mathrm{HT}_{1 \mathrm{~B} / 1 \mathrm{D}}$ receptor agonists, are effective and well tolerated in acute migraine management [7]. They are migraine specific which suggest unique mechanisms of action [8]. Several mechanisms have been suggested, including vasoconstriction of cranial vessels [9] and decreased CGRP release [10]. The triptans reduce CGRP release from stimulated trigeminal neurons in vitro [11], and increased CGRP level normalizes after subcutaneous sumatriptan during migraine [10] and cluster headache attacks [12]. CGRP levels may, however, also normalize spontaneously during attacks of both migraine [13] and cluster headache [14]. These data raise the question whether the CGRP decrease is a contributor to the clinical efficacy of triptans, or merely an epiphenomenon [15].

To examine the mechanisms of sumatriptan action, we have examined whether sumatriptan modulate the baseline 
CGRP levels in vivo, under conditions without trigeminovascular system activation.

It has been suggested that animal studies of CGRP release and neurovascular trigeminal activation could be a good model for investigating antimigraine drugs [16]. To compensate for potential species differences [17], human studies are necessary. In this pharmacological model study, we examined whether activation of the $5-\mathrm{HT}_{1 \mathrm{~B} / 1 \mathrm{D}}$ receptors would result in a decreased CGRP release in healthy human subjects.

The aim of the study was to discover and quantify changes in plasma levels of CGRP before and after subcutaneous injection of sumatriptan in four different vascular compartments; the internal and external jugular, the cubital veins and the radial artery.

\section{Materials and methods}

We recruited 17 healthy subjects for the study (12 M/5 F, mean age 27.5 years, range $21-35$ years). Inclusion criteria were: age 18-35 years, body weight 50-100 kg. Exclusion criteria were: a personal or a family history of migraine or any other type of headache (except episodic tension type headache less than once a week); headache within $24 \mathrm{~h}$ before study start; any daily medication apart from oral contraceptives; pregnancy or breastfeeding; and serious somatic or psychiatric diseases. Human chorionic gonadotropin (hCG) measurements were done in all female patients before start of the experiments. The intake of coffee, tea, cocoa or other methylxanthine containing foods or beverages was not allowed for the last $8 \mathrm{~h}$ before the start of the study.

The study was approved by the Ethics Committee of the County of Copenhagen (KA-20060069), Danish Medicines Agency, the Danish Data Protection Agency and was undertaken in accordance with the Helsinki Declaration of 1964, as revised in Edinburgh in 2000. The study was monitored by the unit for Good Clinical Practice at Copenhagen University Hospital and registered on http://www.clinicaltrials.gov. All subjects gave written informed consent to participate in the study.

\section{Experimental design}

All procedures were performed in a quiet room with a constant temperature of $25^{\circ} \mathrm{C}$, and the subjects rested in the supine position for $30 \mathrm{~min}$ before baseline measurements. Catheters were inserted in the internal (5Fr $200 \mathrm{~mm}$, BD Careflow $^{\mathrm{TM}}$, Becton Dickinson Ltd, Singapore) and external jugular vein, the cubital vein (16G, Optiva 2, Medex Medical Ltd, Rossendale, Great Britain), and the radial artery (20G, arterial cannula, Becton Dickinson, Swindon,
UK). Procedures were carried out under sterile conditions and sonographic guidance (Side Rite IV Ultrasound Scanner, BARD Access Systems, Inc., Salt Lake City, USA) was used to determine the size and location of the internal jugular catheter. The correct placement in the internal jugular vein was confirmed by the marked $\mathrm{O}_{2}$ difference between the internal and external jugular veins, as described in [18]. We chose injectable sumatriptan (GlaxoSmithKline) to reduce inter-patient variability in pharmacokinetics, and because this formulation yields the highest therapeutic gain in migraine patients [19]. Subcutaneous sumatriptan $6 \mathrm{mg}$ was administrated in the right thigh by a medical doctor (JMH). The drug was delivered from the hospital pharmacy.

Blood samples were collected simultaneously from all four catheters starting-10 min before sumatriptan and then 15, 30, 60, and $90 \mathrm{~min}$ after drug administration. Blood samples were collected in ice-chilled tubes (EDTA and aprotinin). Plasma was separated by centrifugation $(1,000 \mathrm{~g})$ at $4^{\circ} \mathrm{C}$ within half an hour, put on ice until completion of the experiment, and then stored at $-30^{\circ} \mathrm{C}$ until shipment for analysis. Samples were coded and CGRP determinations were assayed blindly with respect to timing and vascular compartments, but with all samples of a patient, to minimize the influence of interassay variation. All catheters were flushed with isotonic saline $0.9 \%$ immediately after sampling and emptied immediately before sampling. The subjects were discharged from the hospital $2 \mathrm{~h}$ after injection of sumatriptan.

\section{Plasma concentration of CGRP}

CGRP was analyzed by radio immuno assay (RIA), as previously described [20]. Human $\alpha$-CGRP was obtained from Peninsula Laboratories Europe (St Helens, UK). Standards were prepared in $0.1 \mathrm{~mol} / \mathrm{L}$ phosphate buffer, $\mathrm{pH}$ 7.5 , containing $0.1 \%$ bovine albumin, $0.01 \%$ sodium azide, and 20KIE/ml aprotinin (Trasylol; Bayer). The tracer was made by labeling Tyr (25-37) amide $\alpha$-CGRP (Multiple Peptide Systems, San Diego, CA) by the Iodo-Gen method (Pierce, Rockford, IL). The tracer was purified by HPLC mounted with a C8 column on a Merck-Hitachi system (E. Merck, Darmstadt, Germany). The polyclonal antibodies were raised in rabbits against human $\alpha$-CGRP specific for the amidated C-terminal end of CGRP. It was used in a final dilution of 1:600,000. Incubation was performed at $4^{\circ} \mathrm{C}$ as a non-equilibrium assay with incubation of sample and antibodies for 4 days, followed by 2-day incubation after addition of tracer. Sample volume was $100 \mu \mathrm{l}$, antibodies was $200 \mu \mathrm{l}$, and tracer was $100 \mu \mathrm{l}$. Separation was performed with a solid-phase separation system (SAC-CEL; IDS, Boldon, UK). The detection limit of the assay was less than $1 \mathrm{pmol} / \mathrm{L}$. The intra assay and 
inter assay coefficients of variation were 2 and $7 \%$, respectively. The non-specific binding was less than $4 \%$.

Data analysis and statistics

We used the Kolmogorov-Smirnov Test to determine whether the data were normally distributed. Results were normally distributed and presented as mean \pm SEM. Calculation of sample size was based on the detection of a difference in CGRP before and after sumatriptan injection at $5 \%$ significance with $80 \%$ power. Therefore, a change in CGRP of $10 \mathrm{pmol} / \mathrm{L}$ in the external jugular vein after subcutaneous sumatriptan was considered clinically significant, based on earlier reports [10]. We assumed that the CGRP analysis had a $8 \%$ inter-individual variation [20], and estimated that 11 subjects should be included [21], but we increased the sample size to 16 subjects since this is statistically more desirable and to have the possibility to compensate for drop-outs.

Repeated-measure ANOVA was used to assess change between groups over time, with catheters as the between subjects factor and time of sample as the within subjects factor. We also compared the area under the CGRP concentration curve (AUC) between the different vascular compartments, calculated according to the trapezium rule [22]. We compared the $\mathrm{AUC}_{\mathrm{CGRP}}$, baseline values and changes over time in CGRP levels within each vascular compartment using the one-way ANOVA. We did a posthoc analysis of the difference in CGRP concentration between baseline and the time of maximal sumatriptan concentration, $C_{\max }, 15 \mathrm{~min}$ after injection [23], using a Dunnett $t$ test. We compared the baseline- $C_{\max }$ difference between compartments using the one-way ANOVA. To determine the agreement between the CGRP concentration in the internal and external jugular system, we used the statistical method described by Bland and Altman for comparing paired data [24]. Changes in vascular variables were analyzed using a paired, two-way $t$ test. Baseline was defined as time $-10 \mathrm{~min}$. All analyzes were performed with SPSS for Windows 15.0 (Chicago, Illinois, USA). Five percent $(P<0.05)$ was accepted as significance level.

Vital signs

Arterial blood pressure was measured on the radial arterial catheter (Gabarith $^{\mathrm{TM}}$, single transducer set, Becton Dickinson Ltd, Singapore), monitored continuously and recorded on paper every $15 \mathrm{~min}$. Heart rate and ECG (Cardiofax V, Nihon-Cohden, Japan) was monitored continuously on an LCD screen and recorded on paper every $15 \mathrm{~min}$.

\section{Results}

Sixteen subjects completed the study $(12 \mathrm{M} / 4 \mathrm{~F}$, mean age 27.5 years, range 21-35 years). Due to catheter related problems there were missing values in samples from internal jugular vein ( $n=5$ out of 80$)$, external jugular vein ( $n=26$ out of 80 ) and cubital vein ( $n=13$ out of 80). The missing values were evenly distributed across time points. There were no differences in baseline concentration of CGRP between internal and external jugular vein, cubital vein, and radial artery $(P=0.93)$ (Table 1$)$.

Plasma levels of CGRP after sumatriptan

Repeated-measure ANOVA showed no interaction between catheter and time of sampling and thus no significant difference in CGRP between the four catheters $(P=0.75)$ (Fig. 1). The $\mathrm{AUC}_{\mathrm{CGRP}}$ did not differ between the four vascular compartments $(P=0.78)$. The ANOVA showed that CGRP did not change over time in the four compartments $(P>0.05)$.

The relative change in CGRP from baseline to $C_{\max }$ was $2.5 \% \pm 2.4$ for the internal jugular, $3.3 \% \pm 5.2$ for the external jugular, $-3.8 \% \pm 4.6$ for the cubital vein, and $4.7 \% \pm 4.4$ for the radial artery; $(P>0.05)$ for all variables. The relative changes in CGRP between baseline and $C_{\text {max }}$ did not differ between the four vascular compartments $(P=0.49)$.

Variation between internal and external jugular CGRP concentration

We found a mean difference of $-0.06 \mathrm{pmol} / \mathrm{L}$ between the two compartments, with a variability of the measurements, also called $95 \%$ limits of agreement (1.96 \pm SD about the mean) [24] of $12.1 \mathrm{pmol} / \mathrm{L}$, (Fig. 2).

The vascular effects of sumatriptan

At $C_{\max }$ sumatriptan induced a significant increase in mean arterial blood pressure $(P<0.001)$, of $7.9 \% \pm 1.5$ relative
Table 1 Baseline concentration CGRP (mean \pm SEM)

\begin{tabular}{llllll}
\hline & $\begin{array}{l}\text { Radial artery } \\
(n=16)\end{array}$ & $\begin{array}{l}\text { Internal jugular } \\
\text { vein }(n=15)\end{array}$ & $\begin{array}{l}\text { External jugular } \\
\text { vein }(n=10)\end{array}$ & $\begin{array}{l}\text { Cubital } \\
\text { vein }(n=15)\end{array}$ & $P$ value \\
\hline CGRP $(\mathrm{pmol} / \mathrm{L})$ & $39.3 \pm 2.9$ & $41.9 \pm 3.2$ & $40.3 \pm 4.4$ & $39.3 \pm 2.9$ & 0.93 \\
\hline
\end{tabular}




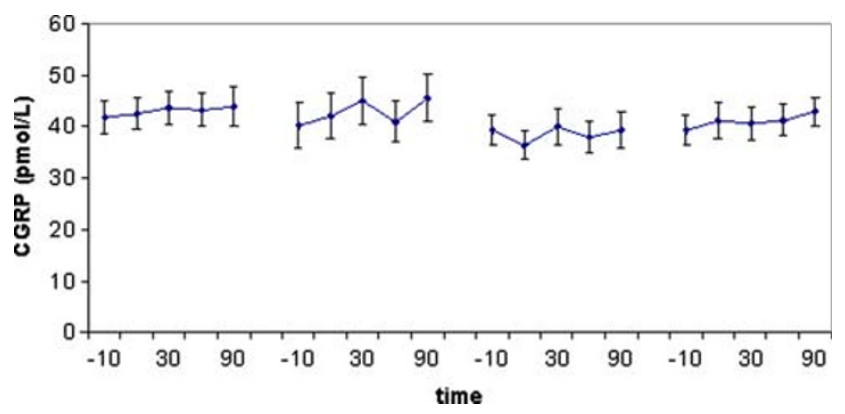

Fig. 1 CGRP concentration before and after sumatriptan. Mean values $( \pm$ SEM) of CGRP $(\mathrm{pmol} / \mathrm{L})$ in the four vascular compartments, before and after $6 \mathrm{mg}$ subcutaneous sumatriptan. There were no differences between the four compartments at baseline $(P=0.93)$

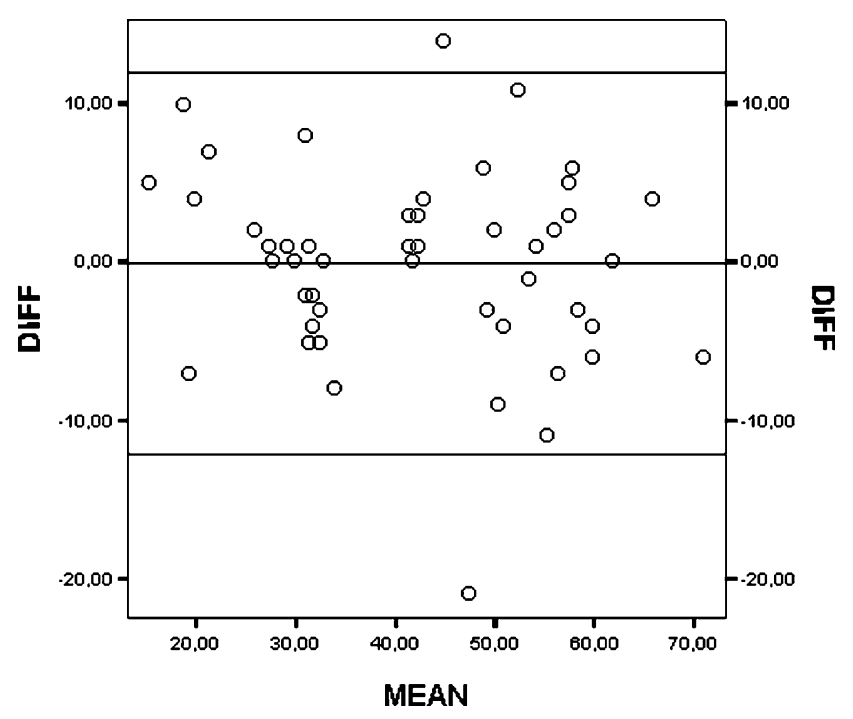

Fig. 2 A graphic presentation of the difference in CGRP between the intra and extracranial circulation. Bland-Altman analysis of the CGRP concentration in the internal and external jugular vein. Mean difference was $-0.06 \mathrm{pmol} / \mathrm{L}$ with a precision of \pm 12.12 . Lines delineate mean and the $95 \%$ limits of agreement (mean $\pm 2 \mathrm{SD}$ )

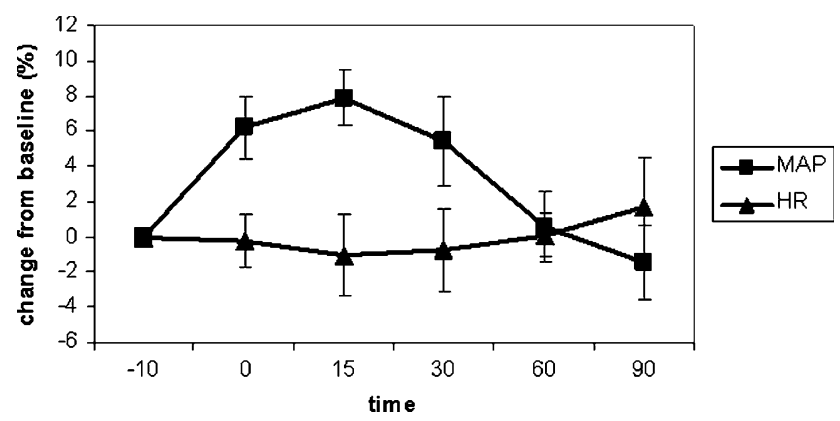

Fig. 3 The vascular effects of sumatriptan. Changes in mean arterial blood pressure (MAP) and heart rate (HR) after sumatriptan $($ mean \pm SEM) to baseline, but did not change heart rate $(P>0.05)$ (Fig. 3).

\section{Adverse events}

The following adverse events were reported: tightness and pressure of the neck and body $(n=10)$, headache $(n=8)$ and a feeling of warmth $(n=5)$. These adverse events occurred shortly after administration, and resolved mostly spontaneously within $30 \mathrm{~min}$. No other periprocedural complications occurred. We will discuss this result in relation to CGRP and the anti-migraine action of triptans.

\section{Discussion}

The major outcome of the present study is that subcutaneous administration of sumatriptan did not change the levels of circulating CGRP in the intra or extracranial circulation in healthy volunteers.

\section{Origin and physiological role of circulating CGRP}

The origin of circulating CGRP is not known in details, but in rats, the major part of circulating CGRP is released from perivascular nerve terminals [25] and CGRP in primary afferents can be released both peripherally and centrally $[26,27]$. In rats, the $5-\mathrm{HT}_{1 \mathrm{D}}$ receptors are found on trigeminal nociceptors [28] and colocalized with CGRP in trigeminal ganglion neurons [29]. CGRP might modulate nociceptive transmission [30] and nociceptor function depends on the sensitivity to CGRP [31].

CGRP is a potent vasodilator [32] in both cerebral and extracerebral arteries [33] and CGRP is broadly distributed in the nervous system [34] including the trigeminovascular system [35].

In the resting state, CGRP is present in plasma in both healthy volunteers [20]. This could be due to leakage after localized release rather than to specific systemic function [36], and CGRP does not affect the resting vascular tone [37]. In pathological settings CGRP might, however, play a role in maintaining vascular tone [38]. This was reported for subarachnoid haemorrhage [39].

\section{Triptans and CGRP}

Stimulation of the trigeminal system causes releases CGRP in vitro [26] resulting in cranial vasodilatation [10] and sensory activation [40].

CGRP is increased in external jugular blood during cluster headache [12]. Migraine attacks and trigeminovascular activation have also been reported to cause a CGRP increase in internal [13] and external jugular blood [3], but 
not in peripheral blood, which could suggest a localized activity in cranial vascular afferent pathways [3]. A larger study could not reproduce the ictal CGRP increase [41], and increased CGRP concentration in migraineurs interictally [42] also obscures the exact role of CGRP in the pathophysiology of primary headaches.

Triptans may lower the CGRP concentration in animals $[26,43]$ and in nitric oxide induced headache [44] and spontaneous migraine attacks in humans [45]. The antimigraine effect of the triptans might therefore be due to inhibited release of CGRP [3]. This effect might be mediated via the $5 \mathrm{HT}_{1}$-receptor, because antagonists to this receptor blocks the CGRP attenuating effect of sumatriptan [43]. The CGRP decrease was, however, present only in patients whose headache improved [10, 44]. It is therefore possible that CGRP levels may decrease spontaneously during migraine attacks [13].

In the present study, we report that sumatriptan does not affect CGRP levels in humans under baseline conditions. This is similar to in vitro results in cultured trigeminal neurons where sumatriptan decreased CGRP secretion from chemically stimulated sensory neurons, but not the basal secretion rate [11]. The concentration of sumatriptan required for this in vitro inhibition was higher than the estimated sumatriptan plasma concentration in patients, and the cell culture showed a higher rate of CGRP-positive cells than seen in vivo [11]. Another drug, topiramate, used for migraine prophylaxis, had also no effect on basal CGRP released from trigeminal neurons, but did decrease CGRP secretion from stimulated trigeminal neurons [46].

The CGRP response to antimigraine drugs could, therefore, rely on activity in the trigeminovascular system. The kinase-phosphatase balance might control CGRP secretion and this balance might be altered during trigeminal activation [11]. Phosphatase activity is increased by sumatriptan, and phosphatase inhibition blocks the inhibitory effect of sumatriptan on stimulated CGRP release [11]. If sumatriptan affects the phosphatases only in activated trigeminal neurons, it could explain the apparent lack of effect in this study, with normal subjects.

In an animal study, triptans effectively prevented the induction of sensitization in central trigemino vascular neurons but not in meningeal nociceptors [47], which could indicate that triptans act on presynaptic $5-\mathrm{HT}_{1 \mathrm{~B} / 1 \mathrm{D}}$ receptors on the central terminals of meningeal nociceptors. Furthermore, it has been reported that the effects of CGRP in the meninges, including meningeal vasodilatation, are insufficient to activate or sensitize meningeal nociceptors [48]. This points toward a central site of action for the migraine-promoting role of CGRP. Triptans might, however, bind to presynaptic $5-\mathrm{HT}_{1 \mathrm{~B} / 1 \mathrm{D}}$ receptors on trigeminal sensory fibers or trigeminal ganglion cells and inhibit nerve activity and hence reduce peripheral CGRP release [49].
Triptans might also interfere with CGRP signaling more centrally $[47,50,51]$, but sumatriptan is hydrophilic and does not easily penetrate the blood brain barrier (BBB) [52]. After BBB disruption, however, sumatriptan alters trigeminal evoked activity in animal studies [53]. If CGRP is mainly released from intra cerebral sources, sumatriptan will inhibit such a release only if the BBB is disrupted [54] which might be the case during migraine attacks [55]. In the present study, the BBB would prevent sumatriptan from reaching the central sites of CGRP release. Clinical efficacy of triptans are however, not directly linked to lipophilicity [7] and the lacking brain penetration of sumatriptan can therefore only partly explain the lack of effect of sumatriptan in the present study. Sumatriptan had pronounced effect on blood pressure (Fig. 3), an effect comparable with earlier results [28] indicating that the drug was present in relevant doses.

Does sumatriptan have specific intracranial effects on CGRP?

Triptans cause greater vasoconstriction in cranial arteries compared to coronary vessels [56], and may thus be selective vasoconstrictors of cerebral arteries [57]. Sumatriptan causes vasoconstriction of the dural vessels [58], but acts only as a weak vasoconstrictor of the superficial temporal artery [59]. This could reflect a different action of sumatriptan on intra and extracerebral vessels.

It has been suggested that blood from the internal jugular vein specifically reflects intracranial neurotransmitter physiology, whereas the external jugular blood can express neurotransmitter and biochemical variations in both intra and extracranial cerebral structures [13].

We therefore explored whether sumatriptan had different effect on the CGRP concentration in blood from the internal and the external jugular system. We found excellent agreement between the levels of CGRP in the internal and the external jugular veins (Fig. 2), with a mean difference of $-0.06 \mathrm{pmol} / \mathrm{L}$ between the two compartments. Under normal circumstances the internal jugular vein is considered to be the most important pathway for venous blood returning from the brain [60]. It thus seems likely that sumatriptan has a similar lack of effect on CGRP in these two vascular beds.

\section{Methodological considerations}

CGRP released from trigeminal afferents is a marker of trigeminovascular activation and can be measured in both experimental animals and humans. The objective of our study was to examine the effect of sumatriptan on plasma levels of CGRP, not to determine whether the drug was effective in migraine treatment. We therefore conducted 
this experiment as a pharmacological model study in healthy volunteers. The model used in the present study might be used for the study of other important signaling molecules in both healthy volunteers and potentially in migraine patients.

Previous studies show a large variation in the CGRP levels. The baseline CGRP levels in this study are larger than jugular levels during migraine [41] but comparable with peripheral CGRP levels during migraine [3]. We have used the same assay as in [41], to minimize interassay variation. CGRP plasma levels show considerable variability [61]. The main objective of our study was to compare CGRP before and after sumatriptan rather than the absolute values, thereby minimizing variability as a potential methodological problem. We drew blood simultaneously from all four catheters thereby eliminating temporal variability as a source of bias. Even though the missing values from the external jugular vein were high $(32.5 \%)$, we have data from 11 subjects, which is sufficient according to our power calculation.

\section{Concluding remarks}

Sumatriptan did not change the levels of circulating CGRP in the intra or extracerebral circulation in healthy volunteers. This speaks against a direct CGRP-reducing effect of sumatriptan in vivo in humans when the trigemino vascular system is not activated.

Acknowledgments The authors acknowledge important contributions to the manuscript from Dr. Saurabh Gupta and Professor Jes Olesen. The authors thank Ms. Alice Rudbøl and Ms. Inger Sassersen for analyzing the blood samples, and the unit for Good Clinical Practice at Copenhagen University Hospital for monitoring the study. This article was supported by The Foundation for Research in Neurology, the University of Copenhagen, the Danish Headache Society and The Lundbeck Foundation for Neurovascular Signalling (LUCENS).

Conflict of interest None.

\section{References}

1. May A, Goadsby PJ (1999) The trigeminovascular system in humans: pathophysiologic implications for primary headache syndromes of the neural influences on the cerebral circulation. J Cereb Blood Flow Metab 19(2):115-127

2. Pietrobon D (2005) Migraine: new molecular mechanisms. Neuroscientist 11(4):373-386

3. Goadsby PJ, Edvinsson L, Ekman R (1990) Vasoactive peptide release in the extracerebral circulation of humans during migraine headache. Ann Neurol 28(2):183-187

4. Goadsby PJ, Edvinsson L, Ekman R (1988) Release of vasoactive peptides in the extracerebral circulation of humans and the cat during activation of the trigeminovascular system. Ann Neurol 23(2):193-196
5. Arulmani U et al (2004) Calcitonin gene-related peptide and its role in migraine pathophysiology. Eur J Pharmacol 500(1-3): 315-330

6. Ho TW et al (2008) Randomized controlled trial of an oral CGRP receptor antagonist, MK-0974, in acute treatment of migraine. Neurology 70(16):1304-1312

7. Ferrari MD et al (2002) Triptans (serotonin, 5-HT1B/1D agonists) in migraine: detailed results and methods of a meta-analysis of 53 trials. Cephalalgia 22(8):633-658

8. Ahn AH, Basbaum AI (2005) Where do triptans act in the treatment of migraine? Pain 115(1-2):1-4

9. Humphrey PP, Feniuk W (1991) Mode of action of the antimigraine drug sumatriptan. Trends Pharmacol Sci 12(12):444446

10. Goadsby PJ, Edvinsson L (1993) The trigeminovascular system and migraine: studies characterizing cerebrovascular and neuropeptide changes seen in humans and cats. Ann Neurol 33(1): $48-56$

11. Durham PL, Russo AF (1999) Regulation of calcitonin generelated peptide secretion by a serotonergic antimigraine drug. J Neurosci 19(9):3423-3429

12. Goadsby PJ, Edvinsson L (1994) Human in vivo evidence for trigeminovascular activation in cluster headache. Neuropeptide changes and effects of acute attacks therapies. Brain 117(Pt 3): 427-434

13. Sarchielli $P$ et al (2000) Nitric oxide metabolites, prostaglandins and trigeminal vasoactive peptides in internal jugular vein blood during spontaneous migraine attacks. Cephalalgia 20(10):907918

14. Fanciullacci M et al (1995) Increase in plasma calcitonin generelated peptide from the extracerebral circulation during nitroglycerin-induced cluster headache attack. Pain 60(2):119-123

15. Humphrey PP, Goadsby PJ (1994) The mode of action of sumatriptan is vascular? A debate. Cephalalgia 14(6):401-410 discussion 393

16. De Vries P, Villalon CM, Saxena PR (1999) Pharmacological aspects of experimental headache models in relation to acute antimigraine therapy. Eur J Pharmacol 375(1-3):61-74

17. Sauerstein K et al (2000) Electrically evoked neuropeptide release and neurogenic inflammation differ between rat and human skin. J Physiol 529(Pt 3):803-810

18. Wienecke $\mathrm{T}$ et al (2008) Sumatriptan does not affect arteriovenous oxygen differences in jugular and cubital veins in normal human subjects. Cephalalgia 28(10):1081-1085

19. Tfelt-Hansen P, De Vries P, Saxena PR (2000) Triptans in migraine: a comparative review of pharmacology, pharmacokinetics and efficacy. Drugs 60(6):1259-1287

20. Schifter S (1991) Circulating concentrations of calcitonin generelated peptide (CGRP) in normal man determined with a new, highly sensitive radioimmunoassay. Peptides 12(2):365-369

21. Campbell MJ, Julious SA, Altman DG (1995) Estimating sample sizes for binary, ordered categorical, and continuous outcomes in two group comparisons. Bmj 311(7013): 1145-1148

22. Matthews JN et al (1990) Analysis of serial measurements in medical research. Bmj 300(6719):230-235

23. Lacey LF, Hussey EK, Fowler PA (1995) Single dose pharmacokinetics of sumatriptan in healthy volunteers. Eur J Clin Pharmacol 47(6):543-548

24. Bland JM, Altman DG (1986) Statistical methods for assessing agreement between two methods of clinical measurement. Lancet 1(8476):307-310

25. Zaidi $M$ et al (1986) The origin of circulating calcitonin generelated peptide in the rat. J Endocrinol 110(1):185-190

26. Buzzi MG et al (1991) Dihydroergotamine and sumatriptan attenuate levels of CGRP in plasma in rat superior sagittal sinus 
during electrical stimulation of the trigeminal ganglion. Neuropharmacology 30(11):1193-1200

27. Levine JD, Fields HL, Basbaum AI (1993) Peptides and the primary afferent nociceptor. J Neurosci 13(6):2273-2286

28. Potrebic $S$ et al (2003) Peptidergic nociceptors of both trigeminal and dorsal root ganglia express serotonin 1D receptors: implications for the selective antimigraine action of triptans. J Neurosci 23(34):10988-10997

29. Ma QP, Hill R, Sirinathsinghji D (2001) Colocalization of CGRP with $5-\mathrm{HT} 1 \mathrm{~B} / 1 \mathrm{D}$ receptors and substance $\mathrm{P}$ in trigeminal ganglion neurons in rats. Eur J Neurosci 13(11):2099-2104

30. Biella $\mathrm{G}$ et al (1991) Facilitatory role of calcitonin gene-related peptide (CGRP) on excitation induced by substance P (SP) and noxious stimuli in rat spinal dorsal horn neurons. An iontophoretic study in vivo. Brain Res 559(2):352-356

31. Mogil JS et al (2005) Variable sensitivity to noxious heat is mediated by differential expression of the CGRP gene. Proc Natl Acad Sci USA 102(36):12938-12943

32. Brain SD et al (1985) Calcitonin gene-related peptide is a potent vasodilator. Nature 313(5997):54-56

33. Edvinsson L et al (1987) Peptide-containing nerve fibers in human cerebral arteries: immunocytochemistry, radioimmunoassay, and in vitro pharmacology. Ann Neurol 21(5):431-437

34. van Rossum D, Hanisch UK, Quirion R (1997) Neuroanatomical localization, pharmacological characterization and functions of CGRP, related peptides and their receptors. Neurosci Biobehav Rev 21(5):649-678

35. Gulbenkian S, Uddman R, Edvinsson L (2001) Neuronal messengers in the human cerebral circulation. Peptides 22(6):9951007

36. Brain SD, Grant AD (2004) Vascular actions of calcitonin generelated peptide and adrenomedullin. Physiol Rev 84(3):903-934

37. Petersen KA et al (2005) The CGRP-antagonist, BIBN4096BS does not affect cerebral or systemic haemodynamics in healthy volunteers. Cephalalgia 25(2):139-147

38. Girgis SI et al (1985) Calcitonin gene-related peptide: potent vasodilator and major product of calcitonin gene. Lancet 2(8445):14-16

39. Juul $\mathrm{R}$ et al (1995) Alterations in perivascular dilatory neuropeptides (CGRP, SP, VIP) in the external jugular vein and in the cerebrospinal fluid following subarachnoid haemorrhage in man. Acta Neurochir (Wien) 132(1-3):32-41

40. Edvinsson L (2004) Blockade of CGRP receptors in the intracranial vasculature: a new target in the treatment of headache. Cephalalgia 24(8):611-622

41. Tvedskov JF et al (2005) No increase of calcitonin gene-related peptide in jugular blood during migraine. Ann Neurol 58(4):561568

42. Ashina $M$ et al (2000) Evidence for increased plasma levels of calcitonin gene-related peptide in migraine outside of attacks. Pain 86(1-2):133-138

43. Eltorp CT, Jansen-Olesen I, Hansen AJ (2000) Release of calcitonin gene-related peptide (CGRP) from guinea pig dura mater in vitro is inhibited by sumatriptan but unaffected by nitric oxide. Cephalalgia 20(9):838-844

44. Juhasz $G$ et al (2005) Sumatriptan causes parallel decrease in plasma calcitonin gene-related peptide (CGRP) concentration and migraine headache during nitroglycerin induced migraine attack. Cephalalgia 25(3):179-183

45. Sarchielli $P$ et al (2006) Clinical-biochemical correlates of migraine attacks in rizatriptan responders and non-responders. Cephalalgia 26(3):257-265

46. Durham PL, Niemann C, Cady R (2006) Repression of stimulated calcitonin gene-related peptide secretion by topiramate. Headache 46(8):1291-1295

47. Levy D, Jakubowski M, Burstein R (2004) Disruption of communication between peripheral and central trigeminovascular neurons mediates the antimigraine action of $5 \mathrm{HT} 1 \mathrm{~B} / 1 \mathrm{D}$ receptor agonists. Proc Natl Acad Sci USA 101(12):4274-4279

48. Levy D, Burstein R, Strassman AM (2005) Calcitonin generelated peptide does not excite or sensitize meningeal nociceptors: implications for the pathophysiology of migraine. Ann Neurol 58(5):698-705

49. Link AS, Kuris A, Edvinsson L (2008) Treatment of migraine attacks based on the interaction with the trigemino-cerebrovascular system. J Headache Pain 9(1):5-12

50. Buzzi MG, Moskowitz MA (1990) The antimigraine drug, sumatriptan (GR43175), selectively blocks neurogenic plasma extravasation from blood vessels in dura mater. Br J Pharmacol 99(1):202-206

51. Burstein R, Jakubowski M (2004) Analgesic triptan action in an animal model of intracranial pain: a race against the development of central sensitization. Ann Neurol 55(1):27-36

52. Tfelt-Hansen $P$ (2000) The effectiveness of combined oral lysine acetylsalicylate and metoclopramide (Migpriv) in the treatment of migraine attacks. Comparison with placebo and oral sumatriptan. Funct Neurol 15(Suppl 3):196-201

53. Kaube H, Hoskin KL, Goadsby PJ (1993) Inhibition by sumatriptan of central trigeminal neurones only after blood-brain barrier disruption. Br J Pharmacol 109(3):788-792

54. Arulmani U et al (2004) Effects of sumatriptan on capsaicininduced carotid haemodynamic changes and CGRP release in anaesthetized pigs. Cephalalgia 24(9):717-727

55. Gursoy-Ozdemir Y et al (2004) Cortical spreading depression activates and upregulates MMP-9. J Clin Invest 113(10):14471455

56. MaassenVanDenBrink A et al (2000) Craniovascular selectivity of eletriptan and sumatriptan in human isolated blood vessels. Neurology 55(10):1524-1530

57. Edvinsson L et al (2005) Triptan-induced contractile (5-HT1B receptor) responses in human cerebral and coronary arteries: relationship to clinical effect. Clin Sci (Lond) 109(3):335-342

58. Henkes $\mathrm{H}$ et al (1996) Sumatriptan: vasoactive effect on human dural vessels, demonstrated by subselective angiography. Cephalalgia 16(4):224-230

59. Razzaque $\mathrm{Z}$ et al (2002) 5-HT1B-receptors and vascular reactivity in human isolated blood vessels: assessment of the potential craniovascular selectivity of sumatriptan. Br J Clin Pharmacol 53(3):266-274

60. Wilson EM, Halsey JH Jr, Vitek JJ (1972) Validation of jugular venous flow as an index of total cerebral blood flow. Stroke 3(3):300-321

61. Onuoha GN et al (2000) Neuropeptide variability in man. Eur J Clin Invest 30(7):570-577 